\title{
Role of Semenov's Theory of Chain Reactions in the Formation of Modern Concepts on the Processes of Combustion, Explosion, and Detonation of Gases
}

\author{
V. V. Azatyan* \\ Semenov Federal Research Center for Chemical Physics, Russian Academy of Sciences, Moscow, 119991 Russia \\ *e-mail:vylenazatyan@yandex.ru \\ Received October 1, 2020; revised October 1, 2020; accepted October 20, 2020
}

\begin{abstract}
This paper presents the results of the theoretical and experimental studies of ignition, flame propagation, explosion, and detonation of gases, showing that, contrary to previous concepts, chain mechanisms in combustion reactions play a decisive role not only at pressures tens of times lower than atmospheric pressure in the absence of self-heating but also at higher pressures and any temperature conditions. Examples of the chemical control of all these processes are given. At the same time, the results show the outstanding role of N.N. Semenov's theory of chain reactions in the development of the theory of the rates of chemical reactions and combustion processes.
\end{abstract}

Keywords: combustion, explosion, detonation, speed, inhibition, free atoms and radicals, chain reactions

DOI: $10.1134 / \mathrm{S} 1990793121020147$

The outstanding contribution of N.N. Semenov to the science of chemical processes is well known. The discovery of branched-chain processes and the creation of a theory of these reactions $[1-3]$ marked the beginning of a qualitatively new, important stage in the development of the science of kinetics and the mechanism of chemical processes, as well as the science of combustion. The significance of this discovery is not limited to the discovery of patterns and mechanisms of combustion processes that have no analogues. The identification of a fundamentally new factor, different from self-heating, which determines the progressive self-acceleration of reactions and previously unknown critical phenomena in combustion processes, is even more significant. Semenov found that the high rates of chain combustion processes occurring at ultralow concentrations of the initial reagents are determined by the reactions of highly active intermediate particles: free atoms and radicals. In reactions with the initial reagents, these particles are not only regenerated but also multiplied. As a result, branched reaction chains, which provide a progressive self-acceleration of the process even without an increase in temperature, are realized. Soon after the discovery by Semenov of branched-chain reactions using the example of the combustion of phosphorus vapors, S. Hinshelwood also established the chain character of the combustion of hydrogen with oxygen $[4,5]$. In subsequent studies, it was found that many other gases also burn along a chain path at low pressures $[3,6]$. The results presented in this study show that the main provisions of the theory of chain processes are also related to the processes of gas combustion that are important in practice and proceed at high pressures.

Ignition and developing combustion are commonly referred to as the kinetic mode of the increasing selfacceleration of a chemical reaction, leading to high speeds of the process, accompanied by the release of light and heat. Two factors, different in nature, can lead to ignition and combustion: a chain avalanche and self-heating.

\section{CHAIN IGNITION}

The branched-chain processes correspond to the following generalized scheme:

$$
\begin{gathered}
\mathrm{y}+\mathrm{B} \rightarrow 3 \mathrm{x}, \\
3(\mathrm{x}+\mathrm{A}) \rightarrow 3 \mathrm{y}+\mathrm{P},
\end{gathered}
$$

where $\mathrm{x}$ and $\mathrm{y}$ are free atoms and radicals; $\mathrm{A}$ and $\mathrm{B}$ are the initial molecules; and $\mathrm{P}$ is the final product.

An example is the model branched-chain process of hydrogen oxidation, in which atoms and radicalschain carriers (CCs)-are formed, regenerated, and multiply in the following reactions:

$$
\begin{gathered}
\mathrm{H}_{2}+\mathrm{O}_{2}=\mathrm{H}+\mathrm{HO}_{2}, \\
\mathrm{H}+\mathrm{O}_{2}=\mathrm{OH}+\mathrm{O}, \\
\mathrm{OH}+\mathrm{H}_{2}=\mathrm{H}_{2} \mathrm{O}+\mathrm{H}, \\
\mathrm{O}+\mathrm{H}_{2}=\mathrm{OH}+\mathrm{H} .
\end{gathered}
$$


Since the CCs appearing at stages (I)-(III) participate in the development of reaction chains, forming new branches, the chain branches. By the method of partial quasi-stationary concentrations [3], the system of kinetic equations for the concentrations of atoms and radicals is reduced to a single equation relating to the particle whose reactions limit the branching of chains. In this process, this reaction is stage (I). From this scheme it follows that the rate of consumption of $\mathrm{O}_{2}$ is

$$
W=-\frac{d[\mathrm{~B}]}{d t}=w_{0}+k_{1}[\mathrm{~B}] n,
$$

where $w_{0}$ is the reaction rate $(0) ; n$ is the concentration of atoms and radicals (CCs); $k_{1}$ is the effective rate constant of the limiting stage of the process, usually the branching stage; and [B] is the concentration of $\mathrm{O}_{2}$.

The CCs also enter into reactions of formation of low-activity products that are not able to participate in chain combustion. In such reactions, the chain is broken. In the process of hydrogen oxidation, such stages are the adsorption of nitrocellulose and the reaction

$$
\mathrm{H}+\mathrm{O}_{2}+\mathrm{M}=\mathrm{HO}_{2}+\mathrm{M},
$$

if the radical $\mathrm{HO}_{2}$ then adsorbed or enters, for example, into the reaction

$$
\mathrm{HO}_{2}+\mathrm{H}=\mathrm{H}_{2}+\mathrm{O}_{2}
$$

and also reacts with the inhibitor (In), which leads to the chain's termination:

$$
\mathrm{H}+\mathrm{In} \rightarrow \text { Break. }
$$

It follows from the reaction scheme that quantity $n$ changes according to the equation $[2,3]$

$$
\frac{d n}{d t}=w_{0}+(f-g) n=w_{0}+\varphi n,
$$

where $f$ and $g$ are the rate of reproduction or death of the CCs at their unit concentrations equal to

$$
\begin{gathered}
f=2 k_{1}[\mathrm{~B}]=2 k_{1}^{0} \exp \left(-\frac{E_{1}}{R T}\right)[\mathrm{B}], \\
g=k_{\text {In }}[\mathrm{In}]+g_{1} .
\end{gathered}
$$

In the last expression $k_{\ln }$ is the inhibition rate constant, [ln] is the inhibitor's concentration, and $g_{1}$ is the effective total rate constant of the other chain termination reactions.

Obviously, if $g>f$, then a low concentration of active particles is established, determined by the ratio of the rates of their formation by the slow reaction (0) and the death reaction. If $f>g$, i.e., the $\mathrm{CC}$ are multiplying faster than death, then their avalanche multiplication occurs. Accordingly, the consumption of the initial reagents reacting with these active particles also accelerates like an avalanche. Thus, the condition for chain ignition is the inequality $\varphi=f-g>0$.

For a long time, chain combustion was studied only to clarify the features of the chemical mechanism of the chain reactions. For this purpose, the reactions were carried out at pressures many tens and hundreds of times lower than atmospheric pressure, at which combustion is practically not accompanied by selfheating. Therefore, the provisions of the theory of chain combustion is related to isothermal regimes. Based on the theory of chain reactions, all the discovered and predicted regularities of processes in conditions of a rarefied flame were quantitatively explained. Among other things, the conclusion from the theory of superequilibrium concentrations of free atoms and radicals in a flame was confirmed. These active particles in concentrations several orders of magnitude higher than the equilibrium values were detected by the method of electron paramagnetic resonance in the flames of a wide variety of combustible substances [7,8]. Note that the recorded high concentrations of atomic oxygen in the flame of phosphorus vapors were predicted by Semenov in 1934 [2]. The experiments also confirmed the conclusion of the theory about the decisive role of the reactions of the free atoms and radicals in all the laws of propagation of a rarefied flame [7-9]. The processes of combustion of gases, accompanied by self-heating, were considered separately, from the standpoint of only thermal phenomena.

\section{THERMAL IGNITION}

Another factor leading to ignition and combustion is the progressive acceleration of the self-heating of the reaction system. Such combustion is usually called thermal combustion. Semenov [3, 6] showed that thermal ignition is realized if the rate of heat release $\left(q_{+}\right)$is greater than the heat sink rate $\left(q_{-}\right)$, and if, with increasing temperature, the heat released accelerates more than the heat removed:

$$
\begin{aligned}
q_{+} & \geq q_{-}, \\
d q_{+} / d T & \geq d q_{-} / d T .
\end{aligned}
$$

Here $T$ is the temperature and $q_{+}$is equal to the product of the reaction rate $(W)$ and the thermal effect $(\bar{Q})$ :

$$
q_{+}=W \bar{Q} .
$$

Equation (5) is related to self-heating. Relation (6) corresponds to a mode in which the rate of heat released increases with increasing temperature more than the rate of heat removed. The simultaneous fulfillment of conditions (5) and (6) leads to a progressive accumulation of heat in the system and to a more intense acceleration of the reaction in the ignition mode. The theory of combustion, which considers self-heating as the only factor in the self-acceleration of the reaction, is usually called thermal.

Significant self-heating of the combustible mixture at pressures above a few hundredths of atmospheric pressure served as the reason that until recently the combustion of gases at such pressures was considered the result of only self-heating. Until recently, in the 


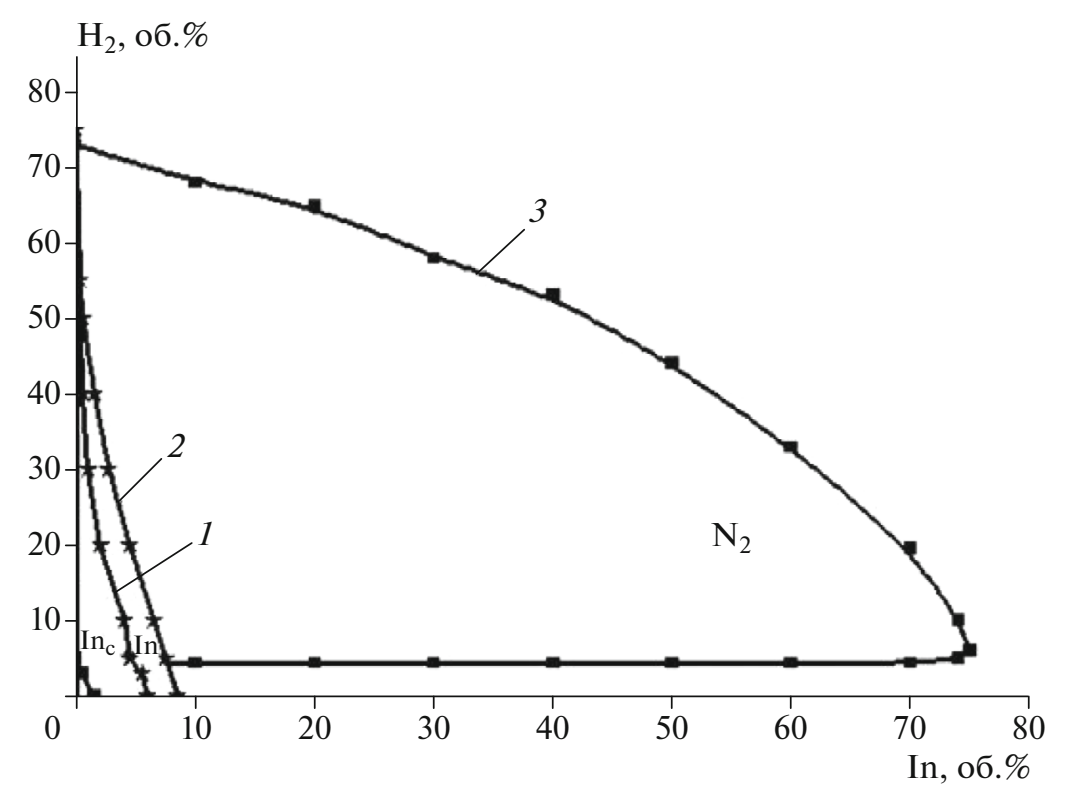

Fig. 1. The areas of ignition of mixtures of hydrogen with air in the presence of isobutene (1), propylene (2), and nitrogen (3).

theory of combustion, explosion, and detonation of gases, as well as in works using the main provisions of this theory, including all articles, fundamental monographs and encyclopedias, the role of reaction chains in combustion at atmospheric pressure was not taken into account, and in some cases it was even denied (see, for example, [10-18]). The chemical process was represented as a hypothetical one-step reaction between the parent molecules. The empirical rate constant is ascribed to such a reaction, determined from the kinetic data of the gross process under the assumption of its first kinetic order. Publications that ignore the chain character of combustion are still encountered [19-21]. It was customary to characterize the temperature dependence of the reaction rate by the Arrhenius law, which, however, refers not to the reaction rate, but to the rate constant. The effective activation energies exceeding $40 \mathrm{kcal} / \mathrm{mol}$ were attributed to hypothetical reactions. These representations made it possible to conditionally describe the course of combustion for the hypothetically assumed design parameters.

Contrary to these concepts, it was shown in a series of theoretical and experimental works [22-27] that intermolecular gas-phase reactions, due to high activation energies, are extremely slow and cannot even provide any significant self-heating, let alone ignition and combustion. Thus, the traditional theory of thermal combustion, based on the model of the reaction of only valence-saturated molecules, cannot even explain the fact of combustion. The apparent agreement of the one-stape model with the experiment is achieved only on the assumption that the combustion reaction proceeds according to the first kinetic order and the use of empirical parameters determined from the data on the research of the same combustion pro- cess. In this case, only one particular pattern is considered. As a result of the arbitrary nature of the choice of the kinetic order the values of most of these empirical parameters do not correspond to their physical meaning.

\section{EVIDENCE OF THE CHAIN NATURE OF REACTIONS IN THE FLAME PROPAGATION, EXPLOSION, AND DETONATION OF GASES}

One of the main proofs of the flow of all modes of combustion of gases by a chain mechanism is the illustration of the impossibility of ignition and combustion when a chain avalanche is excluded using small additives of inhibitors [8, 22-27]. Figure 1 shows that, for example, 3 vol $\%$ of the AKM series of inhibitors (lower olefins) prevents the ignition of any mixtures of hydrogen with air: for any mixture composition to the right of curves 1 and 2 of the concentration limits of such mixtures even strong initiation does not cause ignition. Meanwhile, to prevent ignition by an inert gas, tens of times larger additives are required (curve 3); i.e., a strong dilution of the mixture is necessary. Combustion is blocked by an inhibitor as a result of a quick reaction:

$$
\mathrm{H}+i-\mathrm{C}_{4} \mathrm{H}_{8}=\mathrm{C}_{4} \mathrm{H}_{7}
$$

in which the active particles, $\mathrm{H}$ atoms, are replaced by isobutene radicals that are incapable of participating in the chain reaction of hydrogen combustion.

It was found that ignition has a chain nature also at high initial temperatures [25-27]. The methods developed in these studies make it possible to increase the gas temperature using an incident shock wave (SW) in a fraction of a microsecond by thousands of degrees, 


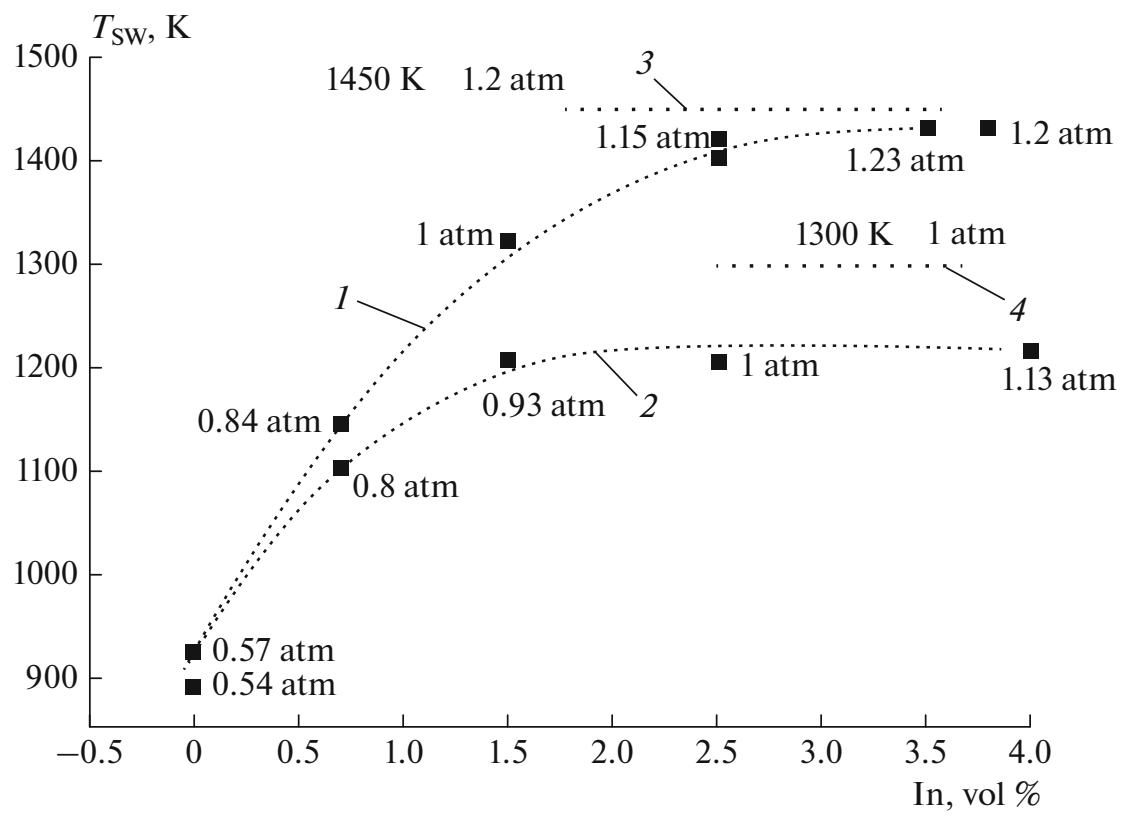

Fig. 2. Effect of propylene (1) and isopropanol vapor (2) for the minimum ignition temperature of hydrogen-air mixtures; $(3,4)$ are the temperature of ignition of propene isopropanol in air.

initiate combustion, and monitor the effect of inhibitors on the initiation and development of combustion at the given initial temperatures, which are uniform over the reactor volume. The gas parameters in the SW front were calculated for each experiment using a wellknown program from the measured SW velocity and the initial mixture's parameters. The signals are recorded by four-beam digital oscilloscopes connected to a computer. The oscillograms also make it possible to follow the transition from combustion to detonation that occurs upon strong initiation. These

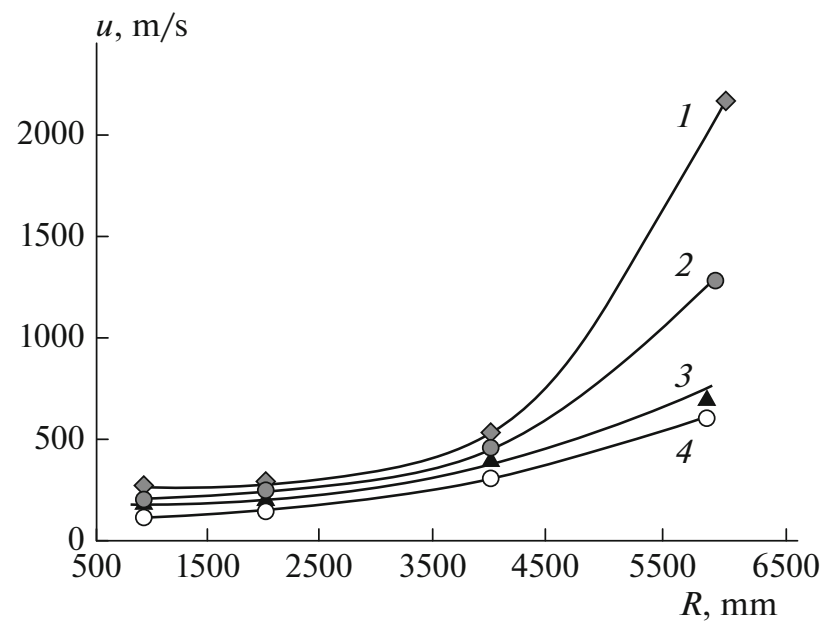

Fig. 3. Change in the speed and flame of a stoichiometric hydrogen-air mixture along the pipe with the following propylene content (\%): (1) 0 ; (2) 0.382 ; (3) 0.764 ; (4) 1.15 . experiments show that even at high initial temperatures and very strong initiation, no ignition occurs in the presence of inhibitors. For example, from Fig. 2 it can be seen that the mixture, which is ignited in the absence of inhibitors at $930 \mathrm{~K}$ and $60 \mathrm{kPa}$, does not ignite even at $1130 \mathrm{~K}$ and a pressure of $84 \mathrm{kPa}$ as a result of the suppression of the chain avalanche by $0.7 \mathrm{vol} \%$ propylene. Neither the glow nor the shortterm pressure rise inherent in ignition is recorded. To ignite a mixture with such a propylene content, it is necessary to increase the rate of the initiating SC and thereby increase the temperature and pressure to $1140 \mathrm{~K}$ and $0.85 \mathrm{~atm}$, respectively. With a further increase in the inhibitor's content, the minimum autoignition temperature becomes even higher (Fig. 2). The critical ignition pressures also increase. Obviously, prevention of ignition also excludes subsequent flame propagation and explosion. Inhibition of a flame propagating at a supersonic speed and prevention of transition to detonation is illustrated in Fig. 3.

Thus, even at very high temperatures, molecular reagents practically do not react directly with each other, and, therefore, in accordance with our conclusion, combustion proceeds only along the chain path. The above-mentioned detection of free atoms and radicals in superequilibrium concentrations in the flame of various combustible substances, as well as a strong excess of the rate constants for the reactions of these active particles with inhibitors in comparison with the chain branching reaction (I), independently confirms this. 


\section{KINETICS OF NON-ISOTHERMAL CHAIN COMBUSTION}

From Eq. (2) it follows that the rate of change in the concentration of active particles is inversely related to the value of their concentration $n$. This means that this value exponentially depends on the difference in the specific rates of branching and breaking of chains: $f-g$. Since the branching rate constant $k_{1}$ with its Boltzmann factor is included in magnitude $f$ in accordance with expression (2), then for $f>g$, magnitude $n$ depends on the temperature according to the exponential law, which is found in the positive exponent [8, 23, 27]:

$$
n=n_{0} \exp \int_{t_{0}}^{t}\left[f_{0} \exp \left(-\frac{E_{p}}{R T}\right)-g\right] d t,
$$

where $t_{0}$ is the time after which the rate of the intermolecular reaction $w$ can be neglected; and $n_{0}$ is the concentration of the $\mathrm{CC}$ at moment $t_{0}$.

From Eqs. (1) and (2), the following dependence of the chain reaction rate on time, temperature, and difference in the rates of branching and termination of the chains is obtained:

$$
\frac{W}{[\mathrm{~B}]}=k_{p} n_{0} \exp \int_{t_{0}}^{t}\left\{2 k_{p}^{0} \exp \left(-\frac{E_{p}}{R T}\right)[\mathrm{B}]-g\right\} d t \text {. }
$$

It can be seen that the speed of the chain process also depends on temperature according to the exponential law, which contains the Boltzmann factor in the positive exponent. Since this law is fulfilled at each given temperature, it is fulfilled during combustion.

Taking into account the chain nature of combustion, all the regularities of the processes in the region of atmospheric and elevated pressures were also explained: the observed very high rates of chain combustion reactions and their strong temperature dependence, which is fundamentally different from the Arrhenius law. Equation (8) containing quantity $g$ and, therefore, the rate of the act of inhibition in the exponent also describes the experimentally observed high efficiency of inhibitors in combustion processes. The slowdown in the reaction is accompanied by a decrease in temperature, which, in turn, leads to a further drop in the rate according to the exponential law, which contains the Boltzmann factor in the positive exponent. Equation (9) also shows that if the inhibitor's concentration is so high that value $g$ is larger than the first term in the integrand of Eq. (8), then the integrand turns out to be negative, and, therefore, a progressive increase in the rate of the process, ignition, and explosion become impossible, i.e., the inhibitor prevents combustion and explosion. At lower concentrations of the inhibitor, the integrand remains positive, but reduced. Accordingly, the reduced reaction speed and flame propagation speed are indicated. This is shown in Fig. 3.
It also follows from Eqs. (4) and (9) that even with a small dilution of the mixture with an inert gas, due to the decrease in the concentration of oxidant $\mathrm{B}$, the reaction and heat release rates decrease exponentially, and the effect of the inhibitors increases. This effect (synergism of an inert gas) was used for a more rational use of inhibitors. Figure 4 shows the results of tests carried out in various organizations to prevent fires and explosions of methane-air mixtures using (curve 1) and without using the phenomenon of synergy (curve 2) [8]. The quantitative agreement of the results of all tests with each other, despite the differences in the shape and volume of the reaction chambers ranging from $3.2 \mathrm{~L}$ to $43 \mathrm{~m}^{3}$ indicates that the inhibitor prevents ignition at the initiation site. It becomes possible to inject the inhibitor locally at the fire site and the source of initiation.

\section{THE DECISIVE ROLE OF THE CHAIN REACTION MECHANISM IN DETONATION AND THE GREAT POSSIBILITIES OF CHEMICALLY CONTROLLING THIS COMBUSTION MODE}

In terms of the kinetics of reactions and gas dynamics, in terms of its role in technology and manifestations in nature, detonation is very different from other combustion modes. From the analysis of the value of the detonation velocity, for example, a stoichiometric mixture of hydrogen with air, and the thickness of the combustion front, it follows that the heating of a gas to the explosion temperature and the consumption of reagents occur in small fractions of a millisecond. In the theory of detonation, which explains in detail the gas-dynamic characteristics of the phenomenon [10, 17, 28, 29], however, the reasons and the chemical mechanism of such high velocities and accelerations were not considered. The participation of reaction chains in detonation and the possibility of its inhibition were considered impossible and not infrequently are currently considered (see, for example, [30, 31]). Until recently, there were also numerical calculations that ignored the participation of reaction chains in detonation, using obviously unrealistic kinetic parameters (see, for example, [21]). Meanwhile, in a series of works [8, 24-27], by preventing the transition of combustion to detonation and the destruction of stationary detonation waves with the help of inhibitors, it was proved that detonation is impossible without a chain avalanche. It was shown that the rates of reactions that ensure the occurrence of an explosion and detonation in millionths of a second are primarily determined by the reactions of rapidly multiplying atoms and radicals with the initial reagents. Only the rapid increase in the concentration of these carriers of the reaction chains in time, enhanced by the growing temperature according to the exponential law, containing the Boltzmann factor in the positive exponent (9), provides the same acceleration of the reaction and heat release. After the 


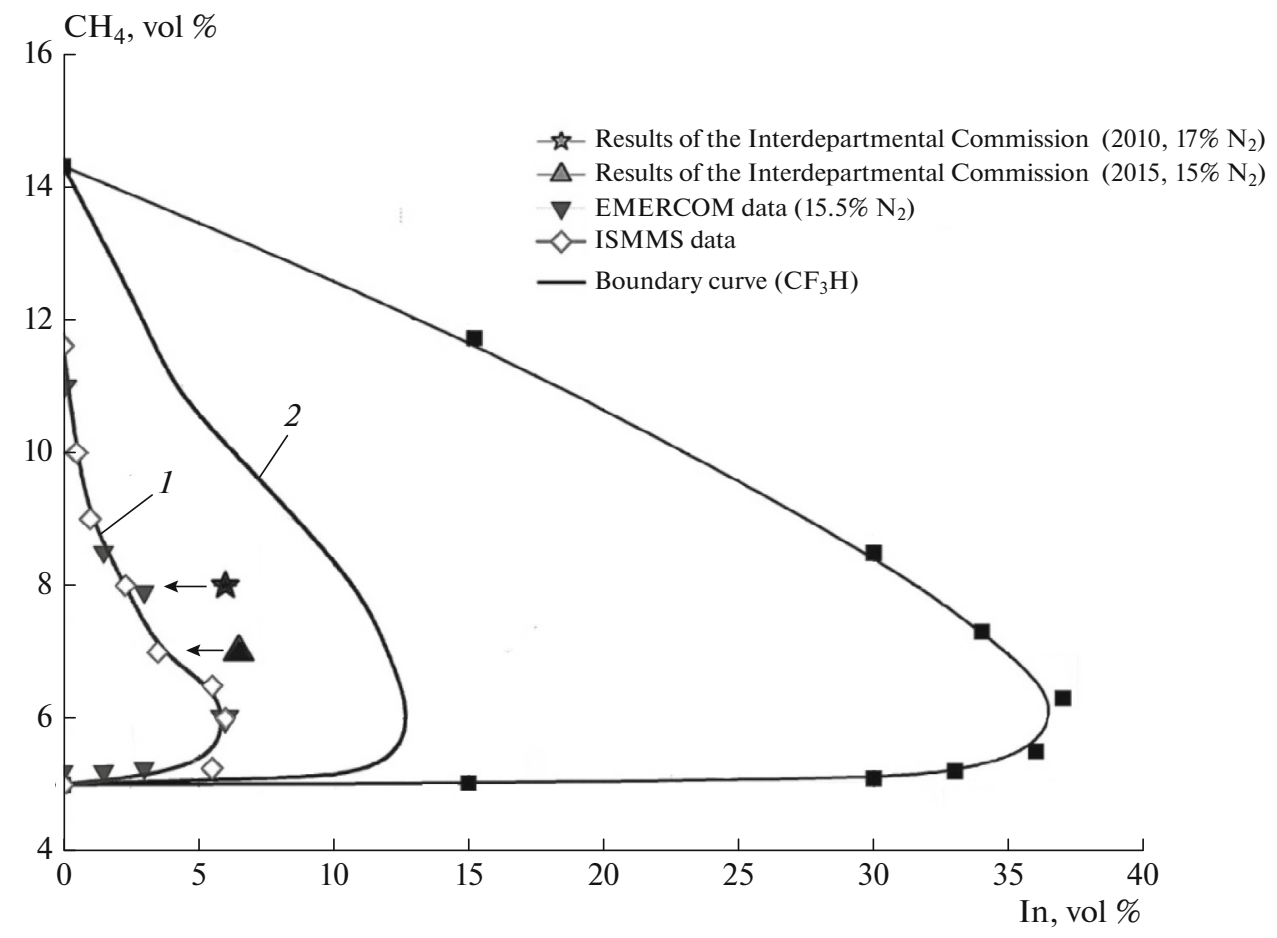

Fig. 4. Narrowing of concentration limits of flame propagation of methane-air mixtures with $\mathrm{CF}_{3} \mathrm{H}$ additives using synergy ( 1 ) and without using synergy (2). Squares are the effect of nitrogen dilution.

characteristic time of the accelerating reaction becomes much shorter than the heat removal time, the process becomes adiabatic and goes into the explosion and detonation mode.

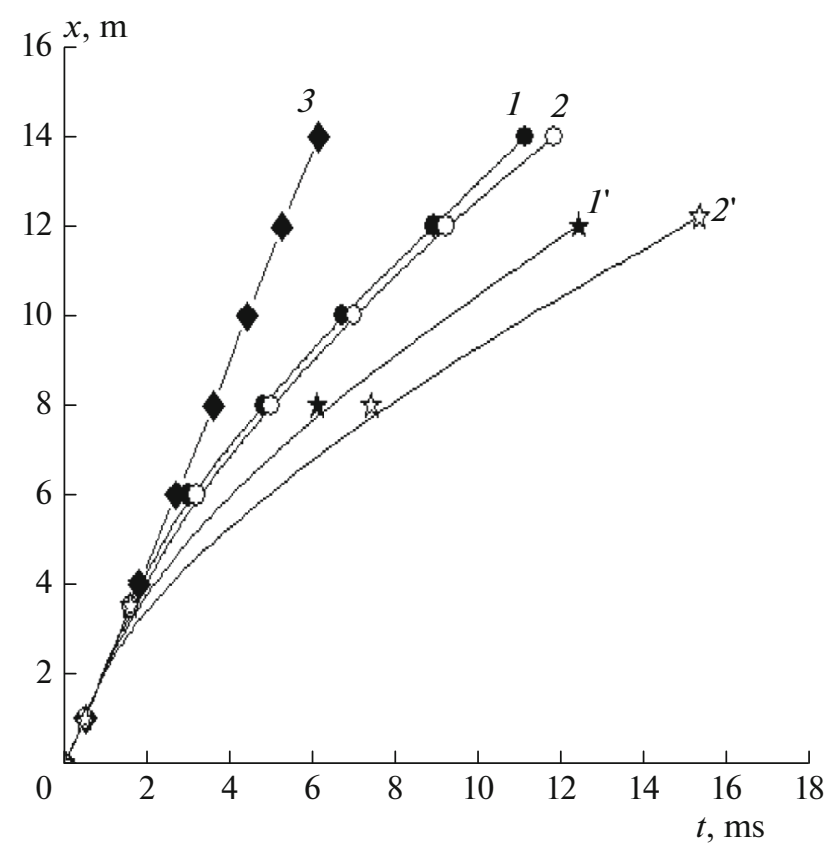

Fig. 5. $x-t$ shock wave diagrams $(1,2)$, combustion fronts $\left(I^{\prime}, 2^{\prime}\right)$, and detonation (3) in a mixture of $45 \% \mathrm{H}_{2}$ in air in the presence of $2.5 \%$ propene $\left(1, l^{\prime}\right)$ and $2.5 \%$ isobutene $\left(2,2^{\prime}\right)$, as well as in the absence of an additive (3) [25].
The exponential nature of the dependence of the rate of the process on the rate of the act of inhibition is the reason not only for the high efficiency of inhibitors but also for the sensitivity of the kinetics of the detonation process to differences in the reactivity of additives. Figure 5 illustrates the dependence of the decay time of stationary detonation on the chemical properties of the inhibitors and members of the same homologous series of olefins [8].

Thus, Semenov's discovery of branched-chain reactions and the subsequent proof of the chain nature of the combustion of gases in the modes of flame propagation, explosion, and detonation made it possible to explain all the main regularities of these processes that had not been explained earlier. It became possible to regulate the following characteristics of the process with the help of additives: the predetonation distance and the delay period of the transition of combustion to detonation, concentration limits and the rate of stationary detonation; it also made it possible to carry out the decay of a stationary wave by acting on these processes with small amounts of additives. A new scientific and technical direction has started developing: the chemical control of combustion, explosion, and detonation of gases.

\section{OPEN ACCESS}

This article is licensed under a Creative Commons Attribution 4.0 International License, which permits use, sharing, adaptation, distribution and reproduction in any medium or 
format, as long as you give appropriate credit to the original author(s) and the source, provide a link to the Creative Commons license, and indicate if changes were made. The images or other third party material in this article are included in the article's Creative Commons license, unless indicated otherwise in a credit line to the material. If material is not included in the article's Creative Commons license and your intended use is not permitted by statutory regulation or exceeds the permitted use, you will need to obtain permission directly from the copyright holder. To view a copy of this license, visit http://creativecommons.org/licenses/by/4.0/.

\section{REFERENCES}

1. N. N. Semenoff, Z. Phys. 46, 109 (1927).

2. N. N. Semenov, Chain Reactions (Goskhimtekhizdat, Leningrad, 1934; United Sci. Tech. Press, 1934).

3. N. N. Semenov, Selected Works (Nauka, Moscow, 2005), Vol. 3 [in Russian].

4. H. W. Thomson and C. N. Hinshelwood, Proc. R. Soc. London, Ser. A 122 (790), 610 (1929).

5. C. N. Hinshelwood and A. T. Williamson, The Reaction between Hydrogen and Oxygen (Oxford Univ. Press, New York, 1934).

6. N. N. Semenov, On Some Problems of Chemical Kinetics and Reactivity (Akad. Nauk SSSR, Moscow, 1958; Elsevier, Amsterdam, 1958).

7. N. N. Semenov, L. B. Soroka, and V. V. Azatyan, Kinet. Katal. 16, 576 (1975).

8. V. V. Azatyan, Chain Reactions in the Processes of Combustion, Explosion and Detonation of Gases (Ross. Akad. Nauk, Moscow, 2017) [in Russian].

9. R. R. Borodulin, E. A. Markevich, V. V. Azatyan, et al., Kinet. Katal. 17 (4), 834 (1976).

10. Ya. B. Zel'dovich, G. I. Barenblatt, V. B. Librovich, et al., Mathematical Theory of Combustion and Explosion (Nauka, Moscow, 1980; Plenum, New York, 1985).

11. V. N. Kondrat'ev and E. E. Nikitin, Chemical Processes in Gases (Nauka, Moscow, 1981) [in Russian].

12. B. Lewis and G. von Elbe, Combustion, Explosions and Flame in Gases (Academic, London, New York, 1987).
13. Chemical Encyclopedy. Combustion (Sov. Entsiklopediya, Moscow, 1988), Vol. 1, p. 594 [in Russian].

14. Physical Encyclopedy. Combustion (Sov. Entsiklopediya, Moscow, 1988), Vol. 1, p. 515 [in Russian].

15. A. G. Merzhanov and B. I. Khaikin, Combustion Wave Theory in Homogeneous Media (OIKhF RAN, Chernogolovka, 1992) [in Russian].

16. D. A. Frank-Kamenetskii, Principles of Macrokinetics: Diffusion and Heat Exchange in Chemical Kinetics (Intellekt, Dolgoprudnyi, 2008; Springer, Heidelberg, 1995).

17. Ya. B. Zel'dovich and A. S. Kompaneets, Theory of Detonation (Gostekhizdat, Moscow, 1955) [in Russian].

18. F. A. Williams, Combustion Theory (CRC, Boca Raton, FL, 1985).

19. S. Tang, M. K. Chernovsky, H. G. Im, et al., Combust. Flame 157, 118 (2010).

20. F. S. Palesskii, R. V. Fursenko, and S. S. Minaev, Combust. Explos., Shock Waves 50, 625 (2014).

21. A. V. Dubrovskii, V. S. Ivanov, A. E. Zangiev, and S. M. Frolov, Russ. J. Phys. Chem. B 10, 469 (2016).

22. V. V. Azatyan, V. I. Bakulev, V. A. Kalkanov, et al., RF Patent No. 1835139 (1992).

23. V. V. Azatyan, Russ. Chem. Rev. 68, 1021 (1999).

24. V. V. Azatyan, G. G. Vagner, and G. K. Vedeshkin, Russ. J. Phys. Chem. A 78, 896 (2004).

25. V. V. Azatyan, V. A. Pavlov, and O. P. Shatalov, Kinet. Catal. 46, 789 (2005).

26. V. V. Azatyan, Russ. J. Phys. Chem. A 85, 1293 (2011).

27. V. V. Azatyan, Kinet. Catal. 61, 319 (2020).

28. V. V. Mitrofanov, Detonation of Homogeneous and Heterogeneous Systems (Sib. Otdel. RAN, Novosibirsk, 2003) [in Russian].

29. Transient Combustion and Detonation Phenomena. Fundamentals and Applications (Torus, Moscow, 2014) [in Russian].

30. A. S. Sokolik, Self-Ignition, Flame and Detonation in Gases (Akad. Nauk SSSR, Moscow, 1960) [in Russian].

31. B. E. Gel'fand, Fiz. Goreniya Vzryva 38 (5), 101 (2002). 